\title{
Evaluation of BIONOR Mono-kits for rapid detection of bacterial fish pathogens
}

\author{
Jesús L. Romalde, Beatriz Magariños, Belen Fouz, Isabel Bandín, \\ Soledad Núñez, Alicia E. Toranzo* \\ Departamento de Microbiología y Parasitología, Facultad de Biología, Universidad de Santiago, \\ E-15706 Santiago de Compostela, Spain
}

\begin{abstract}
The accuracy of the latex agglutination-based BIONOR Mono-kits for the rapid detection of different bacterial fish pathogens was evaluated. In addition, the usefulness of these commercial kits was compared with that of conventional slide agglutination techniques using specific monovalent antisera. The Mono-Yr (for detection of Yersinia ruckeri) and Mono-Va (for Vibrio anguillarum) kits specifically detected the isolates belonging to the serotypes included in the kit's composition (serotypes 01 , O2, and $\mathrm{O} 3$ of each species]. Moreover, the Mono-Va kit was also able to detect some strains of the environmental serotypes of $V$. anguillarum (O4, O5, and O7). The Mono-Pp kit (against Pasteurella piscicida) reacted with all the $P$. piscicida strains included in this study, with no cross-reactions with other bacterial groups. However, non-specific agglutinatıons were observed in both, test and negative control reagents, when isolates of $V$. splendidus or motile Aeromonas were analyzed with the Mono-Va and between some clinical members of the Pasteurellaceae family and the Mono-Pp. The kit for detection of Renibacterium salmoninarum (Mono-Rs) recognized all the strains possessing the predominant p57 antigen, but failed to agglutinate some isolates lacking this surface protein in their cell envelopes. These findings indicate that, although the BIONOR Mono-kits can be easily improved (i.e. including more antigens in heterogeneous groups, changing the inert protein in some of the kits, etc), they are applicable for a rapid screening of the fish pathogens studied. In addition, the kits present an adequate cost-effectiveness ratio, with a simple test performance and interpretation, which make easy their use by aquaculture facilities and laboratories.
\end{abstract}

KEY WORDS: Latex agglutination kits - Yersinia ruckeri Vibrio anguillarum - Pasteurella piscicida . Renibacterium salmoninarum

\section{INTRODUCTION}

Serological procedures, in particular agglutination tests, have been developed in recent years in response to the need for rapid tools for disease diagnosis in aquaculture. With these techniques, not only has the time required for recognition of many of the major infectious diseases been reduced from days to hours, but also the accuracy of diagnosis has been increased.

Since the introduction of a simple slide agglutination test for the identification of Aeromonas salmonicida by Rabb et al. (1964), this procedure has been applied to a

\footnotetext{
- Addressee for correspondence
}

great number of bacterial fish pathogens with few problems of cross-reactivity (Eurell et al. 1979, Toranzo et al. 1987a). However, the appearance of autoagglutinating strains has reduced the use of the slide agglutination tests in some bacterial species. The use of latex agglutination procedures (McCarthy 1975) has solved this problem, enabling the identification of smooth and autoagglutinating strains.

Recently, a Norwegian diagnostic company (BIONOR AS) developed latex agglutination kits (Monokits) for the rapid detection of several fish pathogens with the aim of avoiding the fairly laborious and timeconsuming classical methods for diagnosis of fish diseases and to facilitate the identification of pathogens in the field. 
The purpose of this study was to evaluate the specificity and sensitivity of these Mono-kits in the detection of some important pathogens causing disease problems in continental and marine aquaculture over the world, namely Yersinia ruckeri, Vibrio anguillarum, Pasteurella piscicida and Renibacterium salmoninarum. In addition, the usefulness of these commercial kits was compared with that of conventional slide agglutination techniques using specific antisera produced in our laboratory.

\section{MATERIALS AND METHODS}

Bacterial strains. To evaluate the accuracy of the 4 BIONOR Mono-kits, a collection of 85 strains from 4 bacterial species pathogenic for fish, with different geographical origin and host species, and representative of the different serotypes within each species, were employed. Accordingly, this study included 25 Yersinia ruckeri isolates, 26 Vibrio anguillarum isolates, 18 Pasteurella piscicida strains, and 16 Renibacterium salmoninarum isolates. The identity of each isolate was confirmed with standard biochemical procedures (Toranzo et al. 1987b, Fouz et al. 1990, Romalde et al. 1990, Romalde \& Toranzo 1991, Bandín et al. 1992, Magariños et al. 1992). In addition, in order to assess the specificity of the detection kits, 52 isolates belonging to species taxonomically related to those cited above were also utilized: Yersinia intermedia, $Y$. kristensenii, $Y$. enterocolitica, Vibrio ordalii, V. tubiashii, V. damsela, V. splendidus, V. pelagius, Aeromonas hydrophila, A. sobria, A. caviae, A. salmonicida subsp. salmonicida, atypical A. salmonicida, Pasteurella multocida, P. haemolytica, Actinobacillus pleuropneumoniae, Haemophilus parasuis, Enterococcus sp., E. seriolicida, E. fecalis, Lactococcus garvieae, Carnobacterium piscicola and Corynebacterium aquaticum (Tables 1 to 4 ).

Representatives of Yersinia and Aeromonas genera were routinely grown in tryptic soy agar (TSA; Difco Lab., Detroit, MI, USA). For the culture of the halophilic Vibrio species and Pasteurella piscicida strains this medium was supplemented with $1 \%$ (wt/vol) $\mathrm{NaCl}$. With the exception of Renibacterium salmoninarum, the remaining strains were cultured in Blood Agar (bioMerieux, Madrid, Spain). The incubation was conducted at 22 or $37^{\circ} \mathrm{C}$ (depending on the strain) over 24 to $48 \mathrm{~h}$. In the case of the fastidious R. salmoninarum strains, Mueller-Hinton agar (Difco) supplemented with $1 \%$ (wt/vol) cysteine (MH-C) was employed, being incubated at $15^{\circ} \mathrm{C}$ for at least $15 \mathrm{~d}$. Stock cultures of the strains were maintained at $-70^{\circ} \mathrm{C}$ in appropriate media with $15 \%$ (vol/vol) glycerol.
Detection systems. BIONOR Mono-kits: These kits utilize latex particles coated with specific polyvalent sheep antisera against various bacterial pathogens such as Yersinia ruckeri (Mono-Ys), Vibrio anguillarum (Mono-Va), Pasteurella piscicida (Mono-Pp), and Renibacterium salmoninarum (Mono-Rs). Mono-Yr and Mono-Va include antisera against 3 strains from the main pathogenic serotypes (O1, O2 and O3) of each species. In the kits Mono-Pp and Mono-Rs, the latex particles are coated with the serum raised against a single strain of the respective species. When incubated with microorganisms obtained from a pure culture, the specific antiserum bind to the organism, resulting in an agglutination of the latex particles and thus providing the identity of the bacterial culture. The kits also contain a negative control reagent consisting of monodispersed latex particles coated with non-specific protein.

Kits were used following the manufacturer's instructions. Positive results were recorded only if clear agglutination was seen within $30 \mathrm{~s}$ after mixing the test reagent with the bacteria and if no reaction was observed with the negative control reagent. To estimate the sensitivity of these commercial kits, 10-fold serial dilutions of the antigen preparations were made and the detection limit was expressed as number of bacteria $\mathrm{ml}^{-1}$ needed to obtain agglutination.

Conventional slide agglutination tests: Selected strains of Yersinia ruckeri, Vibrio anguillarum, Pasteurella piscicida and Renibacterium salmoninarum were employed as antigens for immunization. The isolates chosen on the basis of the serological heterogeneity of the bacterial species were: Y. ruckeri 11.4 (serotype O1), 11.29 (serotype O2) and 1533/87 (serotype O3); $V$. anguillarum R 82 (serotype O1), RV 22 (serotype O2) and ATCC 43307 (serotype O3); $P$. piscicida DI 21; $R$. salmoninarum ATCC 33209 (possessing the p57 antigen) and R 1 (lacking the p57 surface protein) (Tables 1 to 4 ).

Immunization was performed following basically the procedures of Toranzo et al. (1987b). Briefly, suspensions of formalin-killed bacteria (approximately $10^{9}$ cells $\mathrm{ml}^{-1}$ ) were injected intravenously in New Zealand rabbits in consecutive daily doses of $0.25,0.5,1.0$, and $2 \mathrm{ml}$ followed by a single $1.5 \mathrm{ml}$ injection $2 \mathrm{wk}$ later. One week after the last injection, the rabbits were bled by cardiac puncture. Blood was allowed to clot and the separated sera were stored at $-30^{\circ} \mathrm{C}$ until used.

Agglutination assays were performed by mixing a drop of bacterial suspension of about $10^{9}$ cells $\mathrm{ml}^{-1}$ (antigen preparations) with a drop of antiserum. A distinct agglutination in $30 \mathrm{~s}$. was registered as positive, and a weak agglutination after 5 min was considered as a negative test. Controls were made with saline and serum from non immunized rabbits. Sensitivity was determined as above. 
Somatic ' $O$ ' antigens were prepared as previously described (Toranzo et al. 1987b) by heating the bacterial suspensions at $100^{\circ} \mathrm{C}$ for $1 \mathrm{~h}$ and the agglutination results obtained compared with those of whole cell preparations. duced in our laboratory are compiled in Tables 1 to 4 . The effectiveness achieved by each kit in the detection of the different pathogens, as well as their sensitivities are described below.

\section{RESULTS}

The results obtained in the comparative study of accuracy between the Mono-kits and the conventional slide agglutination test using specific antisera pro-

\section{Yersinia ruckeri}

The Mono-Yr kit (containing particles coated with antisera against the classical serotypes 01,02 and O3) detected all the strains belonging to serotypes $O 1$ and O3 using $24 \mathrm{~h}$ cultures (Table 1). With the exception of

Table 1. Results obtained in the comparative analysis between BlONOR Mono-Yr and slide agglutination test using specific monovalent antisera. In parenthesis: detection limits of the Mono-kit and the specific monovalent antisera as no. bacteria ml ${ }^{-1}$

\begin{tabular}{|c|c|c|c|c|c|c|}
\hline \multirow[t]{2}{*}{ Strain } & \multirow[t]{2}{*}{ Serotype } & \multirow[t]{2}{*}{ Origin } & \multirow[t]{2}{*}{ Mono-Yr } & \multicolumn{3}{|c|}{ Antisera against Yersinia ruckeri strain } \\
\hline & & & & $\begin{array}{c}11.4 \\
\text { (serotype O1) }\end{array}$ & $\begin{array}{c}11.29 \\
\text { (serotype O2) }\end{array}$ & $\begin{array}{c}1533 / 87 \\
\text { (serotype O3) }\end{array}$ \\
\hline \multicolumn{7}{|l|}{ Yersinia ruckeri } \\
\hline NCIMB 1316 & O1 & Rainbow trout, UK & $+\left(10^{8}\right)$ & $+\left(10^{7}\right)$ & - & $+\left(10^{7}\right)$ \\
\hline FP-13 & O1 & Rainbow trout, Spain & $+\left(10^{8}\right)$ & $+\left(10^{7}\right)$ & - & $+\left(10^{8}\right)$ \\
\hline PP-31 & O1 & Rainbow trout, Spain & $+\left(10^{8}\right)$ & $+\left(10^{7}\right)$ & - & $+\left(10^{7}\right)$ \\
\hline$A G-71$ & O1 & Rainbow trout, Spain & $+\left(10^{9}\right)$ & $+\left(10^{7}\right)$ & - & $+\left(10^{7}\right)$ \\
\hline$A G-143$ & O1 & Rainbow trout, Spain & $+\left(10^{9}\right)$ & $+\left(10^{7}\right)$ & - & $+\left(10^{7}\right)$ \\
\hline$Y F-1$ & O1 & Rainbow trout, Spain & $+\left(10^{8}\right)$ & $+\left(10^{7}\right)$ & - & $+\left(10^{7}\right)$ \\
\hline $15 \mathrm{R}$ & 01 & Rainbow trout, Spain & $+\left(10^{8}\right)$ & $+\left(10^{7}\right)$ & - & $+\left(10^{7}\right)$ \\
\hline$A F-1$ & O1 & Water, Spain & $+\left(10^{9}\right)$ & $+\left(10^{7}\right)$ & $(+)^{a}$ & $+\left(10^{8}\right)$ \\
\hline 11.4 & 01 & Rainbow trout, USA & $+\left(10^{9}\right)$ & $+\left(10^{7}\right)$ & $(+)^{a}$ & $+\left(10^{8}\right)$ \\
\hline 11.40 & O1 & Rainbow trout, USA & $+\left(10^{8}\right)$ & $+\left(10^{7}\right)$ & $(+)^{a}$ & $+\left(10^{7}\right)$ \\
\hline $\mathrm{RS} 2$ & $\mathrm{O} 2$ & Rainbow trout, Canada & - & - & $+\left(10^{9}\right)$ & - \\
\hline RS6 & $\mathrm{O} 2$ & Brook trout, Canada & $+\left(10^{10}\right)$ & - & $+\left(10^{9}\right)$ & - \\
\hline 11.29 & $\mathrm{O} 2$ & Chinook salmon, USA & $+\left(10^{8}\right)$ & $(+)^{\mathrm{a}}$ & $+\left(10^{7}\right)$ & - \\
\hline 11.31 & $\mathrm{O} 2$ & Rainbow trout, USA & $+\left(10^{9}\right)$ & - & $+\left(10^{7}\right)$ & - \\
\hline $1622 / 87$ & $\mathrm{O} 2$ & Atlantic salmon, Norway & $+\left(10^{8}\right)$ & - & $+\left(10^{8}\right)$ & - \\
\hline $1638 / 87$ & $\mathrm{O} 2$ & Atlantic salmon, Norway & $+\left(10^{9}\right)$ & - & $+\left(10^{7}\right)$ & - \\
\hline $87 / 09 / 700$ & $\mathrm{O} 2$ & Rainbow trout, UK & $+\left(10^{9}\right)$ & - & $+\left(10^{7}\right)$ & - \\
\hline $1533 / 87$ & $\mathrm{O} 3$ & Atlantic salmon, Norway & $+\left(10^{8}\right)$ & $+\left(10^{8}\right)$ & - & $+\left(10^{7}\right)$ \\
\hline RS54 & $\mathrm{O} 3$ & Brook trout, Canada & $+\left(10^{8}\right)$ & $+\left(10^{7}\right)$ & - & $+\left(10^{7}\right)$ \\
\hline 11.45 & $\mathrm{O} 3$ & Rainbow trout, Australia & $+\left(10^{9}\right)$ & $+\left(10^{7}\right)$ & - & $+\left(10^{7}\right)$ \\
\hline 11.47 & O5 & Rainbow trout, USA & - & - & - & - \\
\hline $\mathrm{RS} 24$ & 05 & Rainbow trout, Canada & - & - & - & - \\
\hline RS25 & 05 & Rainbow trout, Canada & - & - & - & - \\
\hline 11.73 & O6 & Rainbow trout, USA & $+\left(10^{8}\right)$ & - & - & - \\
\hline RS80 & 06 & Rainbow trout, Canada & - & - & - & - \\
\hline \multicolumn{7}{|c|}{ Yersinia intermedia } \\
\hline IP-16835 & & Water, Italy & - & - & - & - \\
\hline \multicolumn{7}{|c|}{ Yersinia kristensenii } \\
\hline IP-16832 & & Water, Italy & - & - & - & - \\
\hline \multicolumn{7}{|c|}{ Yersinia enterocolitica } \\
\hline Ye 8001 & & Human, USA & - & - & - & - \\
\hline \multicolumn{7}{|c|}{ Vibrio anguillarum } \\
\hline $\mathrm{R}-82$ & O1 & Turbot, Spain & - & - & - & - \\
\hline $\mathrm{RV}-22$ & $\mathrm{O} 2$ & Turbot, Spain & - & - & - & - \\
\hline \multicolumn{7}{|c|}{ Aeromonas hydrophila } \\
\hline B-32 & O3 & Rainbow trout, Spain & - & - & - & - \\
\hline $80-\mathrm{A} 1$ & 024 & Rainbow trout, Spain & - & - & - & - \\
\hline
\end{tabular}


the strain RS2, the kit also showed positive results with the serotype $\mathrm{O} 2$ strains, but to obtain a clear reaction it was necessary to subculture the colonies or to perform the tests with $48 \mathrm{~h}$ cultures (data not shown). As expected, on the basis of its composition, the kit was unable to detect Yersinia ruckeri strains of serotypes 05 and 06 . On the other hand, sensitivity assays showed that a minimum concentration of $10^{8}$ bacteria $\mathrm{ml}^{-1}$ is needed in the reaction to get a positive agglutination.

Regarding the conventional slide agglutination test using specific antisera, both anti-serotype $\mathrm{O} 1$ and antiserotype $\mathrm{O} 3$ reacted with all the strains belonging to these 2 serotypes (Table 1). The anti-serotype $\mathrm{O} 2$ serum detected only the isolates of this serotype. In addition, some cross-reactions were observed among serotypes $\mathrm{O} 1$ and $\mathrm{O} 2$ when whole cell preparations were used as antigen, but not with the somatic ' $O$ ' antigens (data not shown). The detection limit of the rabbit. sera anti-Yersinia ruckeri was $10^{7}$ cells $\mathrm{ml}^{-1}$ (Table 1 ).

No cross-reactivity with other environmental and clinical Yersinia species or with other fish pathogens such as Vibrio anguillarum and motile aeromonads was detected by any of the procedures utilized, BIONOR Mono-kits or slide agglutination test.

\section{Vibrio anguillarum}

As in the case of Yersinia ruckeri, this Mono-Va kit contains latex particles coated with antisera against serotypes $\mathrm{O} 1, \mathrm{O} 2$ and $\mathrm{O} 3$ of Vibrio anguillarum. Therefore, the kit was highly effective for the detection of strains of these 3 serotypes. Moreover, it was able to react with some strains of $V$. anguillarum belonging to the environmental serotypes $\mathrm{O} 4, \mathrm{O} 5$ and O7 (Table 2). Non-specific agglutinations occurred in both test and control reagents when Vibrio splendidus and motile Aeromonas strains were tested, but not with other Vibrio species like $V$. damsela or $V$. pelagius. However, no cross-reactions were observed with other related fish pathogens such as Pasteurella piscicida or Aeromonas salmonicida. No differences were observed in the results obtained after 24 or $48 \mathrm{~h}$ of incubation.

The 3 monovalent antisera (against serotypes $\mathrm{O} 1, \mathrm{O} 2$ and 03) employed in the slide agglutination tests, detected specifically the isolates belonging to the homologous serotype. No cross-reactivity was observed with other serotypes of Vibrio anguillarum or with different species of the genera Vibrio and Aeromonas (Table 2).

The sensitivity assays showed, as in the case of Yersinia ruckeri, a difference of about 1 to $2 \log$ units among the titers obtained with the Mono-Va kit and those yielded by our specific antisera. Thus, a bacterial concentration of $10^{8}$ cells $\mathrm{ml}^{-1}$ was needed to obtain a positive reaction with the latex particles while $10^{7}$ bacteria $\mathrm{ml}^{-1}$ was enough to obtain agglutination with the rabbit antisera (Table 2).

\section{Pasteurella piscicida}

The Mono-Pp kit was able to identify all the Pasteurella piscicida strains included in this study (Table 3). Cross-reactions with representative isolates of related species such as Vibrio anguillarum or Aeromonas salmonicida were not detected. Interestingly, although other members of the family Pasteurellaceae did not react with the test reagent, some non-specific agglutinations were elicited for Pasteurella multocida, Haemophilus parasuis and Actinobacillus pleuropnemoniae strains when the nega-

Table 2. Specificity and sensitivity of the BIONOR Mono-Va kit for the detection of Vibrio anguillarum. In parenthesis: detection limits of the Mono-kit and the specific antisera as no. bacteria $\mathrm{ml}^{-1}$

\begin{tabular}{|c|c|c|c|c|c|c|}
\hline Strain & Serotype & Origin & Mono-Va & $\begin{array}{l}\text { Antisera agai } \\
\text { ATCC } 43305 \\
\text { (serotype } 01 \text { ) }\end{array}$ & $\begin{array}{l}\text { inst Vibrio angu } \\
\text { ATCC } 43306 \\
\text { (serotype 02) }\end{array}$ & $\begin{array}{l}\text { uillarum strain } \\
\text { ATCC } 43307 \\
\text { (serotype O3) }\end{array}$ \\
\hline \multicolumn{7}{|c|}{ Vibrio anguillarum } \\
\hline $\operatorname{ATCC} 43305$ & O1 & Rainbow trout, Denmark & $+\left(10^{8}\right)$ & $+\left(10^{7}\right)$ & - & - \\
\hline$R-82$ & 01 & Turbot, Spain & $+\left(10^{8}\right)$ & $+\left(10^{7}\right)$ & - & - \\
\hline $96-\mathrm{F}$ & O1 & Striped bass, USA & $+\left(10^{8}\right)$ & $+\left(10^{7}\right)$ & - & - \\
\hline 775 & 01 & Pacific salmon, USA & $+\left(10^{9}\right)$ & $+\left(10^{7}\right)$ & - & - \\
\hline $\mathrm{SO}-86.3$ & 01 & Pacific salmon, Spain & $+\left(10^{9}\right)$ & $+\left(10^{7}\right)$ & - & - \\
\hline TM-14 & 01 & Rainbow trout, Spain & $+\left(10^{8}\right)$ & $+\left(10^{7}\right)$ & - & - \\
\hline $\mathrm{RG}-111$ & $02 \alpha$ & Turbot, Spain & $+\left(10^{9}\right)$ & - & $+\left(10^{7}\right)$ & - \\
\hline ATCC 14181 & $02 \alpha$ & Brown trout, UK & $+\left(10^{9}\right)$ & - & $+\left(10^{7}\right)$ & - \\
\hline ATCC 19264 & $\mathrm{O} 20$ & Cod, Denmark & $+\left(10^{9}\right)$ & - & $+\left(10^{7}\right)$ & \\
\hline ATCC 43306 & 020 & Cod, Denmark & $+\left(10^{9}\right)$ & - & $+\left(10^{7}\right)$ & - \\
\hline
\end{tabular}


Table 2 (continued)

\begin{tabular}{|c|c|c|c|c|c|c|}
\hline \multirow{2}{*}{ Strain } & \multirow{2}{*}{ Serotype } & \multirow{2}{*}{ Origin } & \multirow{2}{*}{ Mono-Va } & \multicolumn{3}{|c|}{ Antisera against Vibrio anguillarum strain } \\
\hline & & & & $\begin{array}{l}\text { ATCC } 43305 \\
\text { (serotype O1) }\end{array}$ & $\begin{array}{c}\text { ATCC } 43306 \\
\text { (serotype O2) }\end{array}$ & $\begin{array}{l}\text { ATCC } 43307 \\
\text { (serotype O3) }\end{array}$ \\
\hline \multicolumn{7}{|c|}{ Vibrio angusllarum (continued) } \\
\hline $43-\mathrm{F}$ & $\mathrm{O} 2 \beta$ & Striped bass, USA & $+\left(10^{8}\right)$ & - & $+\left(10^{7}\right)$ & - \\
\hline RV -22 & $\mathrm{O} 2 \beta$ & Turbot. Spain & $+\left(10^{8}\right)$ & - & $+\left(10^{7}\right)$ & - \\
\hline $6828 \mathrm{C}$ & $O 2 \beta$ & Turbot, Denmark & $+\left(10^{8}\right)$ & - & $+\left(10^{7}\right)$ & - \\
\hline ATCC 43307 & O3 & Rainbow trout, Denmark & $+\left(10^{9}\right\}$ & - & - & $+\left(10^{7}\right)$ \\
\hline $13 \mathrm{~A} 5$ & O3 & Sea water, Spain & $+\left(10^{3}\right)$ & - & - & $+\left(10^{7}\right)$ \\
\hline PT-493 & 03 & Ayu, Japan & $+\left(10^{8}\right)$ & - & - & $+\left(10^{7}\right)$ \\
\hline RPM-5701 & $\mathrm{O} 3$ & Turbot, Spain & $+\left(10^{8}\right)$ & - & - & $+\left(10^{7}\right)$ \\
\hline 11008 & 03 & Seabass, France & $+\left(10^{9}\right)$ & - & - & $+\left(10^{7}\right)$ \\
\hline ET-208 & $\mathrm{O3}$ & Japanese eel, Japan & $+\left(10^{8}\right)$ & - & - & $+\left(10^{7}\right)$ \\
\hline ATCC 43308 & O4 & Cod, Denmark & $+\left(10^{9}\right)$ & - & - & - \\
\hline ATCC 43309 & O5 & Cod, Denmark & $+\left(10^{10}\right)$ & - & - & - \\
\hline ATCC 43310 & 06 & Cod, Denmark & - & - & - & - \\
\hline ATCC 43311 & 07 & European eel, Denmark & $+\left(10^{9}\right)$ & - & - & - \\
\hline ATCC 43312 & 08 & Cod, Denmark & - & - & - & - \\
\hline $\operatorname{ATCC} 43313$ & 09 & Cod, Denmark & - & - & - & - \\
\hline ATCC 43314 & 010 & Cod, Denmark & - & - & - & - \\
\hline \multicolumn{7}{|l|}{ Vibrio ordalii } \\
\hline NCIMB 2167 & & Pacific salmon, USA & - & - & - & - \\
\hline \multicolumn{7}{|l|}{ Vibrio tubiashii } \\
\hline ATCC 19109 & & Clam, USA & - & - & - & - \\
\hline EX1 & & Oyster, Spain & - & - & - & - \\
\hline \multicolumn{7}{|l|}{ Vibrio damsela } \\
\hline ATCC 33539 & & Angel fish, USA & - & - & - & - \\
\hline RG-91 & & Turbot, Spain & - & - & - & - \\
\hline RM-71 & & Turbot, Spain & - & - & - & - \\
\hline \multicolumn{7}{|c|}{ Vibrio splendidus } \\
\hline ATCC 25914 & & Sea water, USA & $--^{a}$ & - & - & - \\
\hline $\mathrm{RG}-52$ & & Turbot, Spain & $-{ }^{a}$ & - & - & - \\
\hline RM-51 & & Turbot, Spain & $-{ }^{a}$ & - & - & - \\
\hline $\mathrm{RI}-42$ & & Turbot, Spain & $--^{a}$ & - & - & - \\
\hline TR-4 & & Rotipher, Spain & $-{ }^{\mathrm{a}}$ & - & - & - \\
\hline \multicolumn{7}{|l|}{ Vibrio pelagius } \\
\hline ATCC 25916 & & Sea water, USA & - & - & - & - \\
\hline $\mathrm{RG}-165$ & & Turbot, Spain & - & - & - & - \\
\hline RA-21 & & Turbot, Spain & - & - & - & - \\
\hline ST-11 & & Atlantic salmon, Spain & - & - & - & - \\
\hline NF-182 & & Rainbow trout, Spain & - & - & - & - \\
\hline \multicolumn{7}{|c|}{ Aeromonas hydrophila } \\
\hline B-32 & O3 & Rainbow trout, Spain & $-{ }^{a}$ & - & - & - \\
\hline B-35 & $\mathrm{O} 3$ & Rainbow trout, Spain & $--^{\mathrm{a}}$ & - & - & - \\
\hline $80-\mathrm{A} 1$ & $\mathrm{O} 24$ & Rainbow trout, Spain & $-{ }^{*}$ & - & - & - \\
\hline \multicolumn{7}{|c|}{ Aeromonas sobria } \\
\hline P-33 & $\mathrm{O} 3$ & Rainbow trout, Spain & $-{ }^{a}$ & - & - & - \\
\hline \multicolumn{7}{|c|}{ Aeromonas caviae } \\
\hline 1.25 & 03 & Human, USA & - & - & - & - \\
\hline \multicolumn{7}{|c|}{ Aeromonas salmonicida subsp. salmonicida } \\
\hline ATCC 33658 & & Atlantic salmon, USA & - & - & - & - \\
\hline MT-004 & & Atlantic salmon, Scotland & - & - & - & - \\
\hline SEG-10.1 & & Atlantic salmon, Spain & - & - & - & - \\
\hline \multicolumn{7}{|c|}{ Pasteurella piscicida } \\
\hline ATCC 17911 & & White perch, USA & - & - & - & - \\
\hline DI-21 & & Seabream, Spain & - & - & - & - \\
\hline 10831 & & Seabass, France & - & - & - & - \\
\hline MP-7801 & & Yellowtail, Japan & - & - & - & - \\
\hline
\end{tabular}


tive control reagent was utilized and for Pasteurella haemolytica with both test and control reagents. On the other hand, our rabbit antiserum raised against $P$. piscicida DI 21 strain, specifically recognized this bacterial group with no cross-reactions with other species.

The detection limit of the Mono-Pp kit was $10^{10}$ bacteria $\mathrm{ml}^{-1}$. This limit decreased to $10^{7}$ cells

Table 3. Effectiveness of the BIONOR Mono-Pp kit in the detection of Pasteurella piscicida. In parenthesis: detection limits of the Mono-kit and the specific antisera as no. bacteria $\mathrm{ml}^{-1}$

\begin{tabular}{|c|c|c|c|}
\hline Strain & Origin & Mono-Pp & $\begin{array}{c}\text { Antiserum against } \\
\text { Pasteurella piscicida DI-21 }\end{array}$ \\
\hline \multicolumn{4}{|c|}{ Pasterurella piscicida } \\
\hline DI -21 & Seabream, Spain & $+\left(10^{10}\right)$ & $+\left(10^{7}\right)$ \\
\hline DI-71 & Seabream, Spain & $+\left(10^{10}\right)$ & $+\left(10^{7}\right)$ \\
\hline DI-91 & Seabream, Spain & $+\left(10^{10}\right)$ & $+\left(10^{7}\right)$ \\
\hline $\mathrm{B}-32$ & Seabream, Spain & $+\left(10^{10}\right)$ & $+\left(10^{7}\right)$ \\
\hline B- 51 & Seabass, Spain & $+\left(10^{10}\right)$ & $+\left(10^{7}\right)$ \\
\hline$B-52$ & Seabass, Spain & $+\left(10^{10}\right)$ & $+\left(10^{7}\right)$ \\
\hline 10831 & Seabass, France & $+\left(10^{10}\right)$ & $+\left(10^{7}\right)$ \\
\hline IT -1 & Seabream, Italy & $+\left(10^{10}\right)$ & $+\left(10^{7}\right)$ \\
\hline IT -2 & Seabream, italy & $+\left(10^{10}\right)$ & $+\left(10^{7}\right)$ \\
\hline MP-7801 & Yellowtail, Japan & $+\left(10^{10}\right)$ & $+\left(10^{7}\right)$ \\
\hline KP 9006 & Yellowtail, Japan & $+\left(10^{10}\right)$ & $+\left(10^{7}\right)$ \\
\hline EPOY-8803-II & Red grouper, Japan & $+\left(10^{10}\right)$ & $+\left(10^{7}\right)$ \\
\hline P-3333 & Amberjack, Japan & $+\left(10^{10}\right)$ & $+\left(10^{7}\right)$ \\
\hline P. 3334 & Yellowtail, Japan & $+\left(10^{10}\right)$ & $+\left(10^{7}\right)$ \\
\hline AT LIT 2 & Striped bass, Israel & $+\left(10^{10}\right\}$ & $+\left(10^{7}\right)$ \\
\hline AT LIT 18 & Seabream, Israel & $+\left(10^{10}\right)$ & $+\left(10^{7}\right)$ \\
\hline ATCC 17911 & White perch, USA & $+\left(10^{10}\right)$ & $+\left(10^{7}\right)$ \\
\hline ATCC 29690 & Yellowtail, Japan & $+\left(10^{10}\right)$ & $+\left(10^{7}\right)$ \\
\hline \multicolumn{4}{|c|}{ Pasteurella multocida } \\
\hline CECT 902 & Bovine, Spain & $-^{\alpha}$ & - \\
\hline 04041 & Porcine, The Netherlands & $--^{\mathrm{a}}$ & - \\
\hline \multicolumn{4}{|c|}{ Pasteurella haemolytica } \\
\hline CECT 924 & Ovine, Spain & $-^{\prime \prime}$ & - \\
\hline \multicolumn{4}{|c|}{ Haemophilus parasuis } \\
\hline 94388 & Unknown, The Netherlands & $--^{a}$ & - \\
\hline \multicolumn{4}{|c|}{ Actinobacillus pleuropneumoniae } \\
\hline WST 25 & Porcine, The Netherlands & $--^{\mathrm{a}}$ & - \\
\hline \multicolumn{4}{|c|}{ Vibrio anguillarum } \\
\hline $\mathrm{R}-82$ & Turbot, Spain & - & - \\
\hline 775 & Pacific salmon, USA & - & - \\
\hline $96-F$ & Striped bass, USA & - & - \\
\hline $\mathrm{SO}-86.3$ & Pacific salmon, Spain & - & - \\
\hline ATCC 19264 & Cod. Denmark & - & - \\
\hline $\mathrm{RV}-22$ & Turbot, Spain & - & - \\
\hline RG-111 & Turbot, Spain & - & - \\
\hline \multicolumn{4}{|c|}{$\begin{array}{l}\text { Aeromonas salmonicida } \\
\text { subsp. salmonicida }\end{array}$} \\
\hline$S R-19.1$ & Rainbow trout, Spain & - & - \\
\hline SEG-10.1 & Atlantic salmon, Spain & - & - \\
\hline E-8101 & Pacific salmon, Japan & - & - \\
\hline $\mathrm{H}-22 / 77$ & Brown trout, UK & - & - \\
\hline MT-004 & Atlantic salmon, UK & - & - \\
\hline 3.101 & Brook trout, USA & - & - \\
\hline ATCC 33658 & Atiantic salmon, USA & - & - \\
\hline \multicolumn{4}{|c|}{ Atypical Aeromonas salmonicida } \\
\hline 2F9-81/A & Flounder, Finland & - & Autoagglutination \\
\hline $26 \mathrm{~F} 23-21 / 1$ & Flounder, Finland & - & Autoagglutination \\
\hline
\end{tabular}


$\mathrm{ml}^{-1}$ when the rabbit antiserum was employed (Table 3)

\section{Renibacterium salmoninarum}

A similar pattern was observed using the Mono-kit and the conventional slide agglutination test with antiRenibacterium salmoninarum ATCC 33209 (typical strain possessing intact p57 antigen), detecting the majority of $R$, salmoninarum isolates. However, both tests failed to agglutinate 3 strains of this bacterial species that lacked the p57 surface protein, namely R1, K57 and K76 (Table 4). Interestingly, our antiserum raised against $R 1$ strain only detected these 3 isolates.
On the other hand, independently of the procedure performed, no cross-reactions were seen with other Gram-positive pathogens belonging to Corynebacterium species and the group of lactic acid bacteria.

As for the other fish pathogens included in this work, differences in sensitivity of about $1 \log$ unit were observed between the Mono-kit and the specific rabbit antisera (Table 4).

\section{DISCUSSION}

The development of serological procedures in the last decades has been associated with major advances in practically all facets of fish health (Schill et al. 1989).

Table 4. Comparative analysis of the BIONOR Mono-Rs kit and conventional slide agglutination test for detection of Renibacterium salmoninarum. In parenthesis: detection limits of the Mono-kit and the specific antisera as no. bacteria $\mathrm{ml}^{-1}$

\begin{tabular}{|c|c|c|c|c|}
\hline Strain & Origin & Mono-Rs & $\begin{array}{r}\text { Monovalent sera ant } \\
\text { ATCC } 33209\end{array}$ & $\begin{array}{l}\text { Salmoninarum } \\
\quad \mathrm{R}-1\end{array}$ \\
\hline \multicolumn{5}{|c|}{ Renibacterium salmoninarum } \\
\hline ATCC 33209 & Pacific salmon, USA & $+\left(10^{8}\right)$ & $+\left(10^{7}\right)$ & - \\
\hline ATCC 33739 & Brook trout, USA & $+\left(10^{8}\right)$ & $+\left(10^{7}\right)$ & - \\
\hline NCIMB 1111 & Salmonid fish, UK & $+\left(10^{8}\right)$ & $+\left(10^{7}\right)$ & - \\
\hline NCIMB 1113 & Brook trout, USA & $+\left(10^{8}\right)$ & $+\left(10^{7}\right)$ & - \\
\hline MT-239 & Atlantic salmon, UK & $+\left(10^{8}\right)$ & $+\left(10^{7}\right)$ & - \\
\hline MT-251 & Atlantic salmon, UK & $+\left(10^{8}\right)$ & $+\left\{10^{7}\right\}$ & - \\
\hline $\mathrm{MT}-417$ & Atlantic salmon, UK & $+\left(10^{8}\right)$ & $+\left(10^{7}\right)$ & - \\
\hline $\mathrm{MT}-421$ & Atlantic salmon, UK & $+\left(10^{8}\right)$ & $+\left(10^{7}\right)$ & - \\
\hline MT-422 & Atlantic salmon, UK & $+\left(10^{8}\right)$ & $+\left(10^{7}\right)$ & - \\
\hline MT-444 & Atlantic salmon, UK & $+\left(10^{8}\right)$ & $+\left(10^{7}\right)$ & - \\
\hline $\mathrm{MOC}$ & Brook trout, Canada & $+\left(10^{8}\right)$ & $+\left(10^{7}\right)$ & - \\
\hline 466 & Pacific salmon, Canada & $+\left(10^{8}\right)$ & $+\left(10^{7}\right)$ & - \\
\hline RS-92 & Pacific salmon, Spain & $+\left(10^{8}\right)$ & $+\left(10^{7}\right)$ & - \\
\hline $\mathrm{R}-1$ & Salmonid fish, Canada & - & - & $+\left(10^{7}\right)$ \\
\hline$K-57$ & Atlantic salmon, UK & - & - & $+\left(10^{7}\right)$ \\
\hline $\mathrm{K}-76$ & Brook trout, Canada & - & - & $+\left(10^{7}\right)$ \\
\hline \multicolumn{5}{|l|}{ Enterococcus sp. } \\
\hline RA-99.1 & Turbot, Spain & - & - & - \\
\hline RM-207.1 & Turbot, Spain & - & - & - \\
\hline AZ-12.1 & Turbot, Spain & - & - & - \\
\hline AZ-64.1 & Turbot, Spain & - & - & - \\
\hline TW-1 & Rainbow trout, Spain & - & - & - \\
\hline \multicolumn{5}{|c|}{ Enterococcus seriolicida } \\
\hline YT -3 & Yellowtail, Japan & - & - & - \\
\hline SS-91014 & Yellowtail, Japan & - & - & - \\
\hline \multicolumn{5}{|c|}{ Enterococcus faecalis } \\
\hline ATCC 19433 & Human, France & - & - & - \\
\hline \multicolumn{5}{|c|}{ Lactococcus garvieae } \\
\hline NCDO 2155 & Bovine, UK & - & - & - \\
\hline \multicolumn{5}{|c|}{ Carnobacterium piscicola } \\
\hline ATCC 35586 & Cutthroat trout, USA & - & - & - \\
\hline $\mathrm{HB}-425$ & Striped bass, USA & - & - & - \\
\hline PT-3.1 & Rainbow trout, Spain & - & - & - \\
\hline \multicolumn{5}{|c|}{ Corynebacterium aquaticum } \\
\hline ATCC 14665 & Distilled water, USA & - & - & - \\
\hline $\mathrm{RB}-968 \mathrm{BA}$ & Striped bass, USA & - & - & - \\
\hline
\end{tabular}


In particular, those regarding accuracy and time required for diagnosis have been significantly improved. Moreover, the possibility of application of these methods under field conditions is of great importance since few fish farms possess their own diagnostic laboratories on site. However, the non-availability of standardized antigens and antisera of known quality can constitute a limitation since they are important requirements for serological procedures to be of value and results of tests reliable. Commercial reagents are now becoming available for use in monitoring fish health. Among these products, rapid latex test kits for the detection of the most important fish pathogens have been developed in the late 1980s. Since then, few studies on their effectiveness, sensitivity and specificity have been performed (Kusser et al. 1988).

Yersinia ruckeri, the causal agent of Enteric Redmouth (ERM) disease, was initially considered an homogeneous bacterial group (Ewing et al. 1978, O'Leary et al. 1979). Soon, serological variations among strains were demonstrated and 5 serotypes

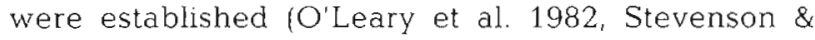
Airdrie 1984, DeGrandis et al. 1988, Davies \& Frerichs 1989). The Mono-Yr kit contains latex particles coated with antisera against the classical serotypes $\mathrm{O} 1, \mathrm{O} 2$ and $\mathrm{O} 3$ which have been mainly associated with the disease outbreaks. Further studies (Romalde et al. 1993) made possible updates in the serological classification of $Y$ ruckeri. Thus, the former serotypes $\mathrm{O} 1$ and $\mathrm{O} 3$ were joined into a new serotype $\mathrm{O} 1$, and the former serotype $\mathrm{O} 2$ was divided into 3 subgroups $(2 \mathrm{a}, 2 \mathrm{~b}$, and $2 c)$. The results obtained here with our specific monovalent antisera raised against representatives of the old serotypes $\mathrm{O} 1, \mathrm{O} 2$ and $\mathrm{O} 3$ supported the new scheme previously reported by the authors (Romalde et al. 1993).

The Mono-Yr kit, as expected, detected all the isolates of the former serotypes 01 and O3. However, and due to the serological heterogeneity demonstrated within the former serotype 02 , some of the representatives of this serotype, particularly those belonging to the subgroup O2a (Romalde et al. 1993), did not react with the latex particles. This fact does not constitute major problems, since isolates of this serological group are very scarce and their relation to fish mortalities and/or disease outbreaks is unclear

For Vibrio anguillarum, 10 serotypes (O1 to 010 ) are defined in the European serotyping scheme (Sorensen \& Larsen 1986), but only serotypes O1, O2 and O3 have been associated with fish mortalities (Toranzo \& Barja $1990,1993)$. Members of the remaining serotypes have been considered environmental strains without pathogenic importance. These environmental serotypes are closely related to other Vibrio species such as V. splendidus and $V$. pelagius (Pazos et al. 1993). The results obtained with the Mono-Va kit revealed a good specificity for the pathogenj.c serotypes 01,02 and 03 . In addition, the detection of some of the environmental serotypes (04, 05 and 07 ) can be explained by the presence of common. minor antigens among these serogroups (Bolinches et al. 1990). On the other hand, some reactions were observed when members of $V$. splendidus and motile Aeromonas were assessed with both test and negative control reagents, even after being subcultured and re-tested 2 d later following the kit instructions. The appearance of these agglutinations, however, fits better (according to the manufacturer's description) with a positive agglutination than with spontaneous agglutination. Therefore, they can be due to non-specific agglutination to the inert protein and/or latex particles. The option of cross-reactivity between related groups with common antigens, although possible in this case, should be ruled out since agglutination also occurred with the negative control reagent.

In contrast, using the conventional slide agglutination test and our monovalent Vibrio anguillarum antisera, no cross-reactions were observed either with environmental serotypes of this species (from $\mathrm{O} 1$ to $\mathrm{O} 10$ ) or with other Vibrio and Aeromonas species.

In contrast to the 2 pathogens already mentioned (Yersinia ruckeri and Vibrio anguillarum), Pasteurella piscicida is a highly homogeneous group, without different serogroups (Magariños et al. 1992). This homogeneity also contrasts with the serological heterogeneity described for other members of the family Pasteurellaceae such as P. multocida and P. haemolytica (Biberstein 1978, Hofacre \& Glisson 1986). Consequently, the Mono-Pp kit exhibited a great specificity for the detection of $P$. piscicida strains with no crossreactions with other bacterial groups. However, agglutinations were displayed only by the negative control reagent when other members of the family such as $P$. multocida, Haemophilus parasuis and Actinobacillus pleuropneumoniae were tested. This fact could be explained by certain reactivity among the inert control protein and these bacterial species. On the other hand, the rabbit serum against the Spanish isolate DI-21 specifically detected all the $P$. piscicida strains without problems of cross-reactions with the other species studied

The major Renibacterium salmoninarum antigen detected in fish sera and tissues in monitoring programs is a protein of $57 \mathrm{kd}$ (Griffiths et al. 1991). This protein can be found as the predominant antigen in both, cell surface and extracellular products (Wiens \& Kaattari 1989). However, instability of this protein has been recently described due to a possible autolytic nature (Griffiths \& Lynch 1991) and the existence of a serin protease activity associated with the cell surface 
(Rockey et al. 1991) The degradation of the major antigen and/or the existence of some strains that never exhibit an intact $57 \mathrm{kd}$ protein could indicate antigenic heterogeneity within $R$. salmoninarum (Bandín et al. 1992). The results obtained in this work with the Mono-Rs latex particles support this hypothesis, since some strains, possessing a degraded p57 protein, were not detected with this kit. The same results were observed when our specific antisera against $R$. salmoninarum were used. The Mono-Rs kit could, therefore, be improved if bacterial antigens of this second group of strains (without $57 \mathrm{kd}$ protein) were also included in the kit. In addition, although the presence of the $57 \mathrm{kd}$ antigen in strains belonging to Corynebacterium aquaticum and Carnobacterium piscicola has been demonstrated by western-blot experiments (Bandin et al. 1993, Toranzo et al. 1993), no cross-reactions were achieved with these 2 species or other members of the lactic acid bacteria by using the Mono-Rs kit or the conventional slide agglutination test with our specific antisera.

Regarding the sensitivity of the commercial Monokits, these always showed higher detection limits than our specific rabbit antisera by at least $1 \log$ unit. Thus, while in the majority of the cases the specific antisera detected concentrations of $10^{7}$ bacteria $\mathrm{ml}^{-1}$, the Mono-kits recognized $10^{8}$ to $10^{9}$ cells $\mathrm{ml}^{-1}$ This difference was significantly higher in Pasteurella piscicida strains for which the kit only detected values of $10^{10}$ bacteria $\mathrm{ml}^{-1}$. These differences in sensitivity can be due to the different host species in which the antisera were raised (sheep in the kits and rabbit in the monovalent antisera) and/or variations in the immunization procedure. Although the Mono-kits showed a great accuracy when applied to bacterial colonies grown in agar plates (for which they were devised), their sensitivity limits hinder the possibility of detection of the pathogens directly in fish fluids or tissues which would represent important time and equipment saving.

In summary, from a practical point of view, we consider that, although the BIONOR Mono-kits can be improved (i.e. by including more antigens in heterogeneous groups, changing the negative control inert protein in some kits, etc), they are applicable for a rapid preliminary screening of the pathogens and disease diagnosis. Moreover, since this test is relatively simple and possesses an adequate cost-effectiveness, it can be used by most aquaculture facilities and laboratories. However, in order to determine the predominant serotype(s) in a given geographical area, and their possible changes in time, specific antisera against the different serotypes within each pathogen must be utilized. This fact is of special importance when performing epidemiological studies and to design effective vaccines
Acknowledgements. The authors acknowledge BIONOR AS (Skien, Norway) for the facilities provided for the study. The kind supply of some strains by multiple donors is greatly appreciated. This work was supported by Grants MAR 91 1133-C02-01 and AGF 94-1360-C03-01 from the Comusion Interministerial de Ciencra y Tecnologia (CICYT) and EUREKA project No. EU-347, between Spain and Norway

\section{LITERATURE CITED}

Bandín I, Santos Y, Barja JL, Toranzo AE (1993) Detection of a common antigen among Renibacterium salmoninarum, Corynebacterium aquaticum, and Carnobacterium piscicola by the western blot technique. J aquat Anim Health 5:172-176

Bandin I, Santos Y. Magariños B, Toranzo AE, Barja JL (1992) The detection of two antigenic groups among Renibacterium salmoninarum strains. FEMS Microbiol Lett 94:105-110

Biberstein EL (1978) Biotyping and serotyping of Pasteurella haemolytica. Meth Microbiol 10:253-268

Bolinches J, Lemos ML, Fouz B, Cambra C, Larsen JL, Toranzo AE (1990) Serological relationships among Vibrio anguillarum strains. J aquat Anim Health 2:21-29

Davies RL, Frerichs GN (1989) Morphological and biochemical differences among isolates of Yersinia ruckeriobtained from wide geographical areas. J. Fish Dis 12:357-365

DeGrandıs SA, Krell PJ, Flett DE, Stevenson RMW (1988) Deoxyribonucleic acid relatedness of serovars of Yersinia ruckeri, the enteric redmouth bacterium. Int $\mathrm{J}$ syst Bacteriol 38:49-55

Eurell TE, Lewis DH, Grumbles LC (1979) Stained bacterial antigens for use in microagglutination procedures. Prog Fish Cult 41:55-57

Ewing WH, Ross AJ, Brenner DJ, Fanning GR (1978) Yersinia ruckeri sp. nov., redmouth (RM) bacterium. Int J syst Bacteriol 28:7-44

Fouz B, Conchas RF, Bolinches J, Romalde JL, Barja JL, Toranzo AE (1990) Relationship among pathogenic Vibrio anguillarum and Vibrio tubiashii with environmental vibrios. In: Perkins FO, Cheng TC (eds) Pathology in marine science. Academic Press, London, p 77-89

Griffiths, SG, Lynch WH (1991) Instability of the major soluble antigen produced by Renibacterium salmoninarum. J Fish Dis 14:55-66

Griffiths SG, Olivier G, Fildes J, Lynch WH (1991) Comparison of western blot, direct fluorescent antibody and dropplate culture methods for the detection of Renibacterium salmoninarum in Atlantic salmon (Salmo salar L.). Aquaculture 97:117-129

Hofacre CL, Glisson JR (1986) A serotypic survey of Pasteurella multocida isolated from poultry. Avian Dis 30: 632-633

Kusser WC, Anderson EP, Newman SG (1988) Development of test kits for bacterial fish pathogens. In: Fish Health Section, American Fisheries Society, Conference handbook. International Fish Health Conference, Vancouver, p 83

Magariños B, Romalde JL, Bandín I, Fouz B, Toranzo AE (1992) Phenotypic, antigenic and molecular characterization of Pasteurella piscicida strains isolated from fish Appl environ Microbiol 58:3316-3322

McCarthy DH (1975) Detection of Aeromonas salmonicida antigen in diseased fish tissue. J gen Microbiol 88: 384-386

O'Leary PJ, Rohovec JS, Fryer JL (1979) A further characterization of Yersinia ruckeri (enteric redmouth bacterium) Fish Pathol 14:71-78 
O'Leary PJ, Rohovec JS, Fryer JL (1982) Serotypes of Yersinia ruckeri and their immunological properties. U.S. Department of the Interior, Technical Paper 6235

Pazos F, Santos Y, Magarıños B, Bandín I, Nuñez S, Toranzo AE (1993) Phenotypic characteristics and virulence of Vibrio anguillarum-related organisms. Appl environ Microbiol 59:2969-2976

Rabb L, Cornick JW, Dermott LA (1964) A macroscopic-slide agglutination test for the presumptive diagnosis of furunculosis in fish. Prog Fish Cult 26:118-120

Rockey DD, Gilkey LL, Wiens GD, Kaattari SL (1991) Monoclonal antibody-based analysis of the Renibacterium salmoninarum p57 protein in spawning chinook and coho salmon. J aquat Anim Health 3:23-30

Romalde JL, Lemos ML, Conchas RF, Bandin I, Toranzo AE (1990) Adhesive properties and other virulence factors in Yersinia ruckeri. In: Perkins FO, Cheng TC (eds) Pathology in marine science. Academic Press, London, p 123-139

Romalde JL, Magarinoos B. Barja JL, Toranzo AE (1993) Antigenic and molecular characterization of Yersinia ruckeri. Proposal for a new intraspecies classification. System appl Microbiol 16:411-419

Romalde JL, Toranzo AE (1991) Evaluation of the API $20 E$ system for the routine diagnosis of the enteric redmouth disease. Bull Eur Ass Fish Pathol 11:147-149

Schill WB, Bullock GL, Anderson DP (1989) Serology. In Austin B, Austin DA (eds) Methods for the micro-biological examination of fish and shellfish. Ellis Horwood Ltd

Responsible Subject Editor: O. Kinne, Oldendorf/Luhe, Germany
Chichester, p 98-140

Sorensen UB, Larsen JL (1986) Serotyping of Vibrio anguillarum. Appl environ Microbiol 51:593-597

Stevenson RMW. Airdrie DW (1984) Serological variation among Yersinia ruckeri stranns. Fish Dis 7:247-254

Toranzo AE, Barja JL (1990) A review of the taxonomy and seroepizootiology of Vibrio anguillarum, with special reference to aquaculture in the northwest of Spain. Dis aquat Org 9:73-82

Toranzo AE, Barja JL (1993) Virulence factors of bacteria pathogenic for coldwater fish. In: Faisal M. Hetrick FM (eds) Annual review of fish diseases, Vol 3. Pergamon Press, Tarrytown, NY, p 5-36

Toranzo AE, Baya AM, Roberson BS, Barja JL, Grimes DJ, Hetrick FM (1987a) Specificity of slide agglutination test for detecting bacterial fish pathogens. Aquaculture 61 $81-97$

Toranzo AE, Romalde JL, Nuñez S, Figueras A, Barja JL (1993) An epizootic in farmed, market size rainbow trout in Spain caused by a strain of Carnobacterium piscicola of unusual virulence. Dis aquat Org 17:87-99

Toranzo AE, Santos Y, Lemos ML, Ledo A, Bolinches J (1987b) Homology of Vibrio anguillarum strains causing epizootics in turbot, salmon and trout reared on the Atlantic coast of Spain. Aquaculture 67:41-52

Wiens GD, Kaattari SL (1989) Monoclonal antibody analysis of common surface protein(s) of Renibacterium salmoninarum. Fish Pathol 24:1-7

Manuscript first received: September 13, 1994 Revised version accepted: November 18, 1994 